$$
\begin{aligned}
& \text { Abbreviations: } \\
& \mathrm{PL}=\text { Plenary; } \mathrm{LO}=\text { Lightning oral } \mathrm{MP}=\text { Moderated poster; } \\
& \mathrm{P}=\text { Poster }
\end{aligned}
$$

*Corresponding authors are underlined.

\section{Plenary Oral Presentations}

\section{PL01}

Multicentre before-after implementation study of the Ottawa subarachnoid hemorrhage strategy

J. J. Perry, MD, MSc, M. L.A. Sivilotti, MD, MSc, M. Emond, MD, MSc, C. M. Hohl, MD, MHSc, H. Lesiuk, MD, J. Sutherland, MEd, M. Khan, MSc, K. Abdulaziz, MSc, G. A. Wells, PhD, I. G. Stiell, MD, MSc, University of Ottawa, Department of Emergency Medicine, Ottawa, ON

Introduction: The Ottawa SAH Rule was developed to identify patients at high-risk for subarachnoid hemorrhage $(\mathrm{SAH})$ who require investigations and the 6-Hour CT Rule found that computed tomography (CT) was $100 \%$ sensitive for SAH 6 hours of headache onset. Together, they form the Ottawa SAH Strategy. Our objectives were to assess: 1) Safety of the Ottawa SAH Strategy and its 2) Impact on: a) CTs, b) LPs, c) ED length of stay, and d) CT angiography (CTA). Methods: We conducted a multicentre prospective before/after study at 6 tertiary-care EDs January 2010 to December 2016 (implementation July 2013). Consecutive alert, neurologically intact adults with a headache peaking within one hour were included. SAH was defined by subarachnoid blood on head CT (radiologists final report); xanthochromia in the cerebrospinal fluid (CSF); $>1 \times 106 / \mathrm{L}$ red blood cells in the final tube of CSF with an aneurysm on CTA. Results: We enrolled 3,669 patients, 1,743 before and 1,926 after implementation, including 185 with SAH. The investigation rate before implementation was $89.0 \%$ (range 82.9 to $95.6 \%$ ) versus $88.4 \%$ (range 85.2 to $92.3 \%$ ) after implementation. The proportion who had CT remained stable $(88.0 \%$ versus $87.4 \%$; $=0.60)$, while the proportion who had LP decreased from $38.9 \%$ to $25.9 \%(\mathrm{p}<0.001)$, and the proportion investigated with CTA increased from $18.8 \%$ to $21.6 \%(\mathrm{p}=0.036)$. The additional testing rate (i.e. LP or CTA) diminishedfrom $50.1 \%$ to $40.8 \%(\mathrm{p}<0.001)$. The proportion admitted declined from $9.8 \%$ to $7.3 \%(\mathrm{p}=0.008)$, while the mean length of ED stay was stable $(6.2+/-4.0$ to 6.4 +/- 4.1 hours; $\mathrm{p}=0.45$ ). For the 1,201 patients with CT 6 hours, there was an absolute decrease in additional testing (i.e. LP or CTA) of $15.0 \%$ (46.6\% versus $31.6 \%$; $<0.001)$. The sensitivity of the Ottawa SAH Rule was $100 \%$ (95\%CI: 98-100\%), and the 6-Hour CT Rule was 95.3\% (95\%CI: 88.998.3) for SAH. Five patients with early CT had SAH with CT reported as normal: 2 unruptured aneuryms on CTA and presumed traumatic LP (determined by treating neurosurgeon); 1 missed by the radiologist on the initial interpretation; 1 dural vein fistula (i.e. non-aneuyrsmal); and 1 profoundly anemic (Hgb 63g/L). Conclusion: The Ottawa SAH Strategy is highly sensitive and can be used routinely when SAH is being considered in alert and neurologically intact headache patients. Its implementation was associated with a decrease in LPs and admissions to hospital.

Keywords: subarachnoid hemorrhage

\section{PL02}

Probiotic regimen for outpatient gastroenteritis utility of treatment (PROGUT) study: a multicenter randomized controlled trial

S. Freedman, MD, CM, MSc, S. Williamson-Urquhart, BSc, K. Farion, MD, S. Gouin, MD, CM, A. Willan, PhD, N. Poonai, MD, MSc, K. Hurley, MD, MHI, P. Sherman, MD, Y. Finkelstein, MD, BSc,
B. Lee, MD, X. Pang, PhD, L. Chui, PhD, D. Schnadower, MD, MPH, J. Xie, MD, MPH, M. Gorelick, MD, MSCE, S. Schuh, MD, University of Calgary, Calgary, AB

Introduction: Gastroenteritis accounts for 1.7 million emergency department visits by children annually in the United States. We conducted a double-blind trial to determine whether twice daily probiotic administration for 5 days, improves outcomes. Methods: 886 children aged 348 months with gastroenteritis were enrolled in six Canadian pediatric emergency departments. Participants were randomly assigned to twice daily Lactobacillus rhamnosus R0011 and Lactobacillus helveticus R0052, $4.0 \times 109 \mathrm{CFU}$, in a 95:5 ratio or placebo. Primary outcome was development of moderate-severe disease within 14 days of randomization defined by a Modified Vesikari Scale score 9. Secondary outcomes included duration of diarrhea and vomiting, subsequent physician visits and adverse events. Results: Moderatesevere disease occurred in $108(26.1 \%)$ participants administered probiotics and $102(24.7 \%)$ participants allocated to placebo (OR 1.06; 95\% CI: 0.77, 1.46; $\mathrm{P}=0.72$ ). After adjustment for site, age, and frequency of vomiting and diarrhea, treatment assignment did not predict moderate-severe disease ( $\mathrm{OR}, 1.11,95 \% \mathrm{CI}, 0.80$ to $1.56 ; \mathrm{P}=0.53)$. In the probiotic versus placebo groups, there were no differences in the median duration of diarrhea $[52.5(18.3,95.8)$ vs. $55.5(20.2,102.3)$ hours; $\mathrm{P}=0.31$, vomiting $[17.7(0,58.6)$ vs. $18.7(0,51.6)$ hours; $\mathrm{P}=0.18$ ], physician visits $(30.2 \%$ vs. $26.6 \%$; OR $1.19 ; 95 \% \mathrm{CI} 0.87$. $1.62 ; \mathrm{P}=0.27)$, or adverse events $(32.9 \%$ vs. $36.8 \%$; OR $0.83 ; 95 \% \mathrm{CI}$ 0.62. 1.11; $\mathrm{P}=0.21$ ). Conclusion: In children presenting to an emergency department with gastroenteritis, twice daily administration of $4.0 \times 109 \mathrm{CFU}$ of a Lactobacillus rhamnosus/helveticus probiotic does not prevent development of moderate-severe disease or improvements in other outcomes measured.

Keywords: probiotic, diarrhea, pediatrics

\section{PL03}

Prophylactic administration of diphenhydramine to reduce neuroleptic side-effects in the acute care setting: a systematic review and meta-analysis

A. Mokhtari, O. Yip, J. Alain, MD, MSc, A. Turgeon, MD, MSc, S. Berthelot, MD, MSc, CHU de Québec Université Laval, Montréal, QC

Introduction: Neuroleptics are commonly used drugs to treat different conditions (e.g. psychosis, migraines) in the acute care setting and the emergency department. Their side effects can be disabling or, worse, fatal. The use of diphenhydramine to prevent those side-effects is widespread, but remains controversial. We performed a systematic review to determine if prophylactic administration of diphenhydramine (PAD) reduces the incidence of neuroleptic side-effects. Methods: Data sources: Medline, Embase, Cochrane Library, PsycInfo and Web of Science were searched. References from reviews that were identified in the search and from included studied were also reviewed for inclusion. Study selection: Randomized controlled trials evaluating any neuroleptic with PAD versus the same neuroleptic alone or with any inactive agent. Primary outcome was incidence of any extra-pyramidal sideeffect. Secondary outcomes were akathisia, usage of rescue medication, subjective restlessness, neuroleptic malignant syndrome, sedation and sedation intensity. Data extraction: Independent reviewers scanned identified citations, extracted data and assessed for risk of bias. Data analysis: Meta-analysis was performed using random effect models. Heterogeneity and quality of evidence were assessed using, respectively, I2 and the GRADE approach. Results: Results: Of 1566 identified citations, nine studies $(n=1436)$ met all eligibility criteria. Four studies 